\title{
Influence of the Microstructure on the Deuterium Retention in Tungsten
}

\author{
A. Manhard ${ }^{*, a}$ K. Schmid, ${ }^{a}$ M. Balden ${ }^{\text {a }}$ W. Jacob ${ }^{\text {a }}$ \\ ${ }^{a}$ Max-Planck-Institut für Plasmaphysik, Boltzmannstraße 2, D-85748 Garching b. \\ München, Germany
}

\begin{abstract}
The microstructure of tungsten samples is systematically modified by recrystallization to investigate the structure dependence of deuterium (D) retention. After a thorough characterization by scanning and transmission electron microscopy, nonrecrystallized samples and samples recrystallized by different degrees are loaded with deuterium in a low-temperature plasma device. The deuterium inventory is measured by nuclear reaction analysis and thermal desorption spectroscopy. The post implantation surface morphology is investigated by scanning electron, optical and atomic force microscopy. The modification by recrystallization allows a wide variation in the crystallite size and has a strong impact on the measured D retention. Both the total amount and the binding state of the retained D are changed.
\end{abstract}

Key words:

PACS: 28.52.Fa, 52.40.Hf, 61.72.-y, 61.72.Ff

\section{Introduction}

Due to its favorable physical properties, such as low erosion yield and high melting temperature, tungsten (W) is foreseen as a plasma-facing material in fusion devices, such as ITER [1]. In these machines the surface material is exposed to large fluxes and fluences of hydrogen isotope ions and energetic neutrals. The hydrogen isotope retention in tungsten is generally considered to be small. Despite substantial efforts to quantify hydrogen retention in tungsten the underlying mechanisms are not yet fully understood $[2,3]$. In particular, the influence of the microstructure can be significant. Therefore, it is

\footnotetext{
* corresponding author

Email address: armin.manhard@ipp.mpg.de (A. Manhard).
} 
necessary to perform dedicated experiments on tungsten samples with a systematic modification and characterization of the microstructure. For this purpose, rolled polycrystalline tungsten samples of the same base material, from a single manufacturing batch, were recrystallized to produce specimens with different grain structures. The grain structure of the non-recrystallized and recrystallized samples was analyzed by scanning electron microscopy (SEM). The dislocation density of the non-recrystallized and recrystallized material was investigated with scanning transmission electron microscopy (STEM). After characterization the samples were implanted with deuterium in a fully quantified low-temperature deuterium plasma with $\mathrm{D}_{3}^{+}$as the dominant ion species. After implantation the D depth profiles in the near surface region (up to $\approx 7 \mu \mathrm{m}$ ) were measured by nuclear reaction analysis (NRA). After NRA, the binding states of the retained D were investigated by Thermal Desorption Spectroscopy (TDS). Great care was taken during transfer and storage of the sample between implantation and the NRA and TDS experiments. Air exposure was avoided where possible to avoid excessive oxidation of the sample. Before and after TDS, the surface morphology of the samples was investigated by optical microscopy. The optical microscopy results were extended by atomic force microscopy and SEM together with focused ion beam techniques for selected details.

This article will first describe the sample preparation and how the structure was modified by recrystallization. Then the D implantation setup and the applied analysis methods, NRA, TDS and microscopy will be described. In the results section the paper will focus on the observed modifications of the $\mathrm{W}$ microstructure and how they relate to the observed variations in the D depth profiles from NRA and binding states from TDS. Also the formation of blisters during $\mathrm{D}$ implantation and their disappearance during degassing of $\mathrm{D}$ in the TDS measurements will be discussed.

\section{Experimental procedure}

The base material for all samples was polycrystalline tungsten ${ }^{1}$ with a guaranteed purity of 99.97 wt.\% rolled to a thickness of $0.8 \mathrm{~mm}$. All samples were cut from sheet metal (thickness $0.8 \mathrm{~mm}$ ) from a single sintering batch to ensure the same initial microstructure and chemical composition. The samples were mechanically polished to a mirror-like finish and cleaned in an ultrasonic bath. Then part of the samples were recrystallized at different temperatures in a graphite furnace with a protective gas atmosphere (Ar for up to $1700 \mathrm{~K}$, He for higher temperatures). They were wrapped in a tungsten foil envelope to prevent carbide formation on the surface. The recrystallization temperatures ranged from 1700 to $2000 \mathrm{~K}$. The holding time was of the order of 20 to

$\overline{1}$ manufactured by PLANSEE Metall GmbH, 6600 Reutte, Austria 
60 minutes and thus always at least twice the time necessary for recrystallization as reported in the literature [4]. Repeated baking at the same temperature showed that the influence of the holding time on the average grain structure was small. Ostwald ripening was occasionally observed, but can be considered a minor effect on these timescales. SEM analysis after recrystallization showed strong formation of small carbide grains on the outside of the envelope, but none on the sample itself. The non-recrystallized samples were degassed for 1 hour at $1200 \mathrm{~K}$ which is well below the recrystallization threshold, but reduces stresses in the samples and removes hydrogen.

After polishing and, for part of the samples, recrystallization, the grain structure of the samples was analyzed by SEM. High grain orientation contrast was achieved by using the annular semiconductor backscattered electron detector (BSE). In order to probe the dislocation density, TEM samples from the nonrecrystallized and the recrystallized material were prepared by electrochemical thinning. They were then analyzed with an electron energy of $30 \mathrm{keV}$ in a high resolution SEM which is also equipped with a STEM detector.

The samples were implanted with deuterium ions in a quantified plasma source [5] which delivers mostly $\mathrm{D}_{3}^{+}$ions. The implantation was performed at $\approx 300 \mathrm{~K}$ with a sample bias of $-100 \mathrm{~V}$ DC. The sample bias together with the plasma potential produces ions with an energy of about $115 \mathrm{eV}$, corresponding to a mean energy of $38 \mathrm{eV}$ per deuteron. The deuteron flux was $9 \times 10^{19} \mathrm{D} \mathrm{m}^{-2} \mathrm{~s}^{-1}$. The non-recrystallized samples were exposed to different fluences between $3 \times$ $10^{23}$ and $6 \times 10^{24} \mathrm{D} \mathrm{m}^{-2}$. The samples recrystallized at 1700 and $2000 \mathrm{~K}$ were implanted together with the non-recrystallized samples during the same plasma exposure at a fluence of $6 \times 10^{24} \mathrm{D} \mathrm{m}^{-2}$.

Previous experiments [6] have shown that prolonged exposure of implanted tungsten samples to ambient air can lead to a large fraction of the deuterium being released during TDS not as $\mathrm{D}_{2}$ molecules, but as heavy water (HDO, $\mathrm{D}_{2} \mathrm{O}$ ). This is attributed to oxide formation on the sample surface during air exposure. The reaction of desorbing deuterium with the surface oxide during TDS analysis then leads to the formation of heavy water. This heavy water fraction is very hard to quantify. In order to suppress surface oxidation, air exposure of the samples was kept to a minimum. After implantation, the samples were stored in a vacuum exsiccator whenever they were not needed for analysis.

To determine the $\mathrm{D}$ amount and depth profile in the near surface region (up to $\approx 7 \mu \mathrm{m})$ the $\mathrm{D}\left({ }^{3} \mathrm{He}, \mathrm{p}\right) \alpha$ nuclear reaction was used. By using energies of the primary ${ }^{3} \mathrm{He}$ beam of 690,1500 and $4000 \mathrm{keV}$ different depth regions can be probed for $\mathrm{D}$ due to the resonant nature of the cross section for this particular nuclear reaction. Based on the shape and intensity of the proton spectrum for each ${ }^{3} \mathrm{He}$ beam energy the depth distribution of $\mathrm{D}$ in sample can be determined 
as described in [7].

The TDS measurements were performed in a dedicated experimental setup located in a low D-background UHV chamber. The sample is heated by electron bombardment and the temperature of the sample is measured by a K-type thermocouple attached to the target. The sample was heated with a linear ramp of $1.4 \mathrm{~K} / \mathrm{s}$ to $1200 \mathrm{~K}$. During heating, HD (mass 3), $\mathrm{D}_{2}$ (mass 4), HDO (mass 19), and $\mathrm{D}_{2} \mathrm{O} / \mathrm{CD}_{4}$ (mass 20) molecules were recorded by a quadruple mass spectrometer (Pfeiffer QMG700). The QMS signal was not calibrated to absolute desorption fluxes. Nonetheless, the desorption spectra and total desorbed D amount can still be compared between samples because identical heating ramps were used.

The surface morphology of all implanted samples was investigated by optical microscopy with differential interference contrast (DIC) before and after TDS. For one of the non-recrystallized samples, markers were applied to the sample surface with the focused ion beam (FIB) of a dual beam SEM/FIB, allowing to investigate exactly the same spot before and after TDS. The dual beam microscope was also used to probe individual blisters that were found on the non-recrystallized samples.

\section{Results and discussion}

The analysis of the grain structure by SEM with BSE contrast showed that the grains on the non-recrystallized material were small with typical dimensions ranging from less than $1 \mu \mathrm{m}$ up to about $2 \mu \mathrm{m}$ (Fig. 1a). Many grains also appeared distorted and were difficult to distinguish. The degassing at $1200 \mathrm{~K}$ did not cause a detectable change of the sample microstructure.

After recrystallization at $1700 \mathrm{~K}$ the grain structure changed significantly. Now many small (approximately 1 to $5 \mu \mathrm{m}$ ) grains were present next to few larger (approximately 10 to $20 \mu \mathrm{m}$ ) grains (Fig. 1b). Note that even the smaller grains are now very clearly visible in the SEM picture and do not appear distorted anymore. Therefore, the sample can be considered fully recrystallized. After recrystallization at $2000 \mathrm{~K}$ the grain size distribution has changed further. Typical grain dimensions are now between 10 and $50 \mu \mathrm{m}$ (see Fig. 1c), and the regions with many small grains, which were present after recrystallization at $1700 \mathrm{~K}$, are no longer present. The sample now comprises much less, but significantly larger grains.

The STEM image of the non-recrystallized sample shows a dense and clearly visible network of dislocations distributed throughout the whole grain (see Fig. 2a). The images of the recrystallized samples (Fig. $2 \mathrm{~b}$ and $2 \mathrm{c}$ ) show only 
very few, faint and often blurred features inside the grains. These are likely due to small dust flakes or chemical residue on the sample surface. Only the grain boundaries are clearly visible.

The deuterium depth distributions determined from NRA for the nonrecrystallized material show a high deuterium concentration in a very narrow region close to the sample surface and a small secondary peak at $\approx 1 \mu \mathrm{m}$ (i.e. far beyond the implantation range of several $\mathrm{nm}$ ). Deeper into the bulk of the sample, the deuterium content decays exponentially as can be seen in Fig. 3. NRA yields an increase of the total retained deuterium amount with fluence as shown in Fig. 4. Comparison with the TDS results (see below) shows the same relative increase of the deuterium inventory.

NRA depth profiling of the recrystallized material revealed the same peaking of the deuterium concentration near the surface as the non-recrystallized material. The exponential decay of the $\mathrm{D}$ concentration at depths well beyond $1 \mu \mathrm{m}$ was also observed. However, in the intermediate region up to $1 \mu \mathrm{m}$, significant changes in the depth profile were observed as Fig. 3 illustrates. In particular the sample recrystallized at $2000 \mathrm{~K}$ showed a considerably lower D concentration in this range, but a very clear secondary peak at $1 \mu \mathrm{m}$. The total amount of deuterium retained in the recrystallized samples is also shown in Fig. 4. The total deuterium inventory of the sample recrystallized at $1700 \mathrm{~K}$ closely matched that of the non-recrystallized sample implanted to the same fluence, while for the one recrystallized at $2000 \mathrm{~K}$ the D inventory was reduced by approximately a factor of 2 . The same relative change was also found by TDS.

TDS showed two $\mathrm{D}_{2}$ release peaks at 520 and $680 \mathrm{~K}$ which are typical for nonrecrystallized tungsten [2]. These release peaks were only rudimentary for the lowest implantation fluence of $3 \times 10^{23} \mathrm{D} \mathrm{m}^{-2}$. For increasing fluence, the $680 \mathrm{~K}$ peak developed first. At $6 \times 10^{24} \mathrm{D} \mathrm{m}^{-2}$, both peaks were clearly identifiable as can be seen in Fig. 5. For the sample recrystallized at $1700 \mathrm{~K}$, only a single broad release peak at approximately $600 \mathrm{~K}$ was observed. For the material recrystallized at $2000 \mathrm{~K}$, the release peak was roughly at the same position. However, it was considerably lower and narrower, so that the total deuterium retention was strongly reduced compared to the other samples exposed to the same fluence.

Optical microscopy with DIC indicated strong blistering of the nonrecrystallized material already after implantation with $3 \times 10^{23} \mathrm{D} \mathrm{m}^{-2}$. The number and average size of blisters increased with fluence. Most blisters have an roughly circular circumference. The diameter of the blisters is up to $\approx 20 \mu \mathrm{m}$. Atomic force microscopy revealed that the blisters have a height of up to $\approx 200 \mathrm{~nm}$. It was discovered that a large fraction of the blisters disappeared after TDS. Fig. 6 shows this for the sample exposed at $3 \times 10^{24} \mathrm{D} \mathrm{m}^{-2}$. 
On this sample, T-shaped markers were cut with the FIB for orientation before TDS. This observation points towards an at least partial elastic deformation due to a high pressure $\mathrm{D}_{2}$ gas filling of subsurface cavities. This assumption is further supported by observations with the dual beam SEM/FIB. Small holes were drilled into the lid of several blisters by the FIB. In regular intervals, the drilling was interrupted in order to image the blister with the SEM. After sufficiently long drilling time, all investigated blisters flattened completely. Cross-sectioning of punctured and collapsed blisters with the FIB and subsequent imaging with the SEM revealed a thin crack system running along grain boundaries at a depth of approximately $1 \mu \mathrm{m}$. On the surface of the recrystallized samples no significant morphology changes, in particular, no blistering was observed after plasma exposure.

\section{Conclusions}

This article reports the investigation of the dependence of the deuterium retention in tungsten on the tungsten microstructure. The crystallite size was varied in a controlled fashion by high temperature recrystallization and subsequently characterized by SEM and STEM microscopy. The STEM investigation also revealed that recrystallization essentially removed most of the dislocations. Investigating the binding states and depth distributions of $\mathrm{D}$ implanted into the samples by TDS and NRA, respectively, revealed that recrystallization had a strong impact on both. In addition, the total fraction of retained D was reduced in the sample recrystallized at $2000 \mathrm{~K}$. In the non-recrystallized samples strong blistering was observed after implantation of low-energy deuterons at a sample temperature of approximately $300 \mathrm{~K}$. Optical microscopy investigation of these blistered samples before and after TDS showed that most of the blisters had disappeared hinting towards elastically deformed gas filled cavities as the dominant blister formation process in these samples. No blistering was observed on the recrystallized samples. In bulk tungsten, D is mainly trapped as an interstitial at crystal defects like dislocations and grain boundaries since the solubility of $\mathrm{D}$ in a perfect $\mathrm{W}$ lattice is extremely low. The comparison of non-recrystallized and recrystallized W clearly shows a correlation between the $\mathrm{D}$ inventory and the density of these defects. In large cavities, i.e. blisters, however, $\mathrm{D}$ is most likely trapped as $\mathrm{D}_{2}$ gas under high pressure, as the disappearance of the blisters after TDS or after puncturing the blister with the FIB suggests. This conclusion is also supported by the by the results presented in $[8]$. 


\section{References}

[1] R. Parker, G. Janeschitz, H. D. Pacher, D. Post, et al., Plasma-wall interactions in ITER, J. Nucl. Mater. 241-243 (1997) 1.

[2] O. V. Ogorodnikova, J. Roth, M. Mayer, Ion-driven deuterium retention in tungsten, J. Appl. Phys. 103 (2008) 034902.

[3] M. Poon, A. A. Haasz, J. W. Davis, Modelling deuterium release during thermal desorption of $\mathrm{D}^{+}$-irradiated tungsten, J. Nucl. Mat. 374 (2008) 390.

[4] W. Espe, Werkstoffkunde der Hochvakuumtechnik, Vol. 1, VEB Deutscher Verlag der Wissenschaften, Berlin, 1960.

[5] A. Manhard, T. Schwarz-Selinger, W. Jacob, Quantifcation of the deuterium ion fluxes from a plasma source, Plasma Sources Sci. Technol. 20 (2011) 015010.

[6] K. Moshkunov, K. Schmid, M. Mayer, V. Kurnaev, Y. Gasparyan, Air exposure and sample storage time influence on hydrogen release from tungsten, J. Nucl. Mater. 404 (3) (2010) 174-177.

[7] M. Mayer, E. Gauthier, K. Sugiyama, U. von Toussaint, Quantitative depth profiling of deuterium up to very large depths, Nucl. Inst. Meth. B 267 (2009) 506.

[8] V. K. Alimov, J. Roth, R. A. Causey, D. A. Komarov, C. Linsmeier, A. Wiltner, F. Kost, S. Lindig, Deuterium retention in tungsten exposed to low-energy, highflux clean and carbon-seeded deuterium plasmas, J. Nucl. Mat. 375 (2008) 192. 


\section{Figure captions}

Fig. 1. Backscatter SEM images of W samples: a) non-recrystallized, b) $1700 \mathrm{~K}$, c) $2000 \mathrm{~K}$. All images were taken at the same magnification $(1000 \times)$.

Fig. 2. Bright field STEM images of W samples: a) non-recrystallized, b) $1700 \mathrm{~K}$, c) $2000 \mathrm{~K}$. All images were taken at the same magnification $(35000 \times)$.

Fig. 3. NRA depth profiles of D retained in non-recrystallized and recrystallized tungsten samples after bombardment with $38 \mathrm{eV} / \mathrm{D}$ at a sample temperature of $300 \mathrm{~K}$.

Fig. 4. Total amount of D retained in non-recrystallized and recrystallized tungsten samples after exposure to a deuterium plasma with $38 \mathrm{eV} / \mathrm{D}$ at a sample temperature of $300 \mathrm{~K}$ as determined by NRA.

Fig. 5. $\mathrm{D}_{2}$ release spectra from non-recrystallized and recrystallized tungsten samples exposed to a fluence of $6 \times 10^{24} \mathrm{D} \mathrm{m}^{-2}$ with $38 \mathrm{eV} / \mathrm{D}$ at a sample temperature of $300 \mathrm{~K}$. The heating rate was $1.4 \mathrm{~K} / \mathrm{s}$ for all samples.

Fig. 6. Surface of non-recrystallized tungsten sample after implantation (a) and after TDS (b). T-shaped markers were applied to the sample surface by FIB.

\section{Figures}

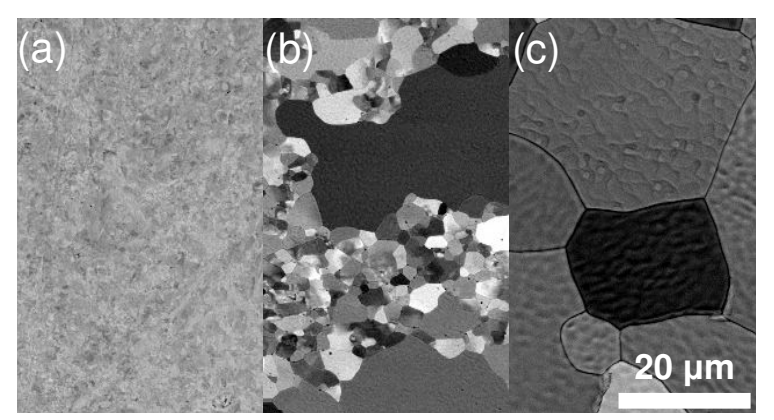

Fig. 1. Backscatter SEM images of W samples: a) non-recrystallized, b) $1700 \mathrm{~K}, \mathrm{c}$ ) 2000 K. All images were taken at the same magnification $(1000 \times)$. 


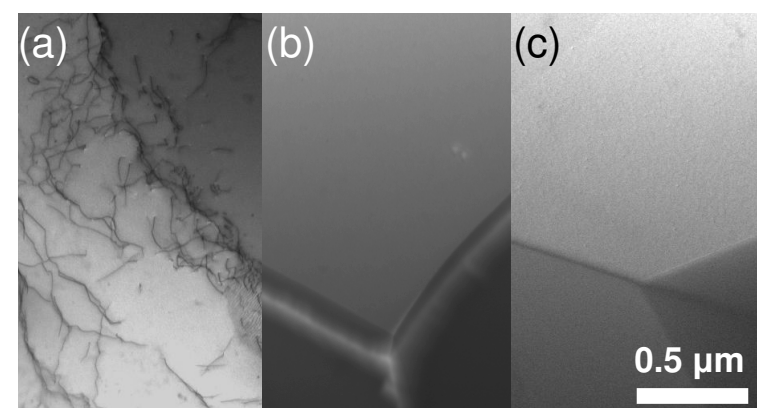

Fig. 2. Bright field STEM images of W samples: a) non-recrystallized, b) $1700 \mathrm{~K}$, c) $2000 \mathrm{~K}$. All images were taken at the same magnification $(35000 \times)$.

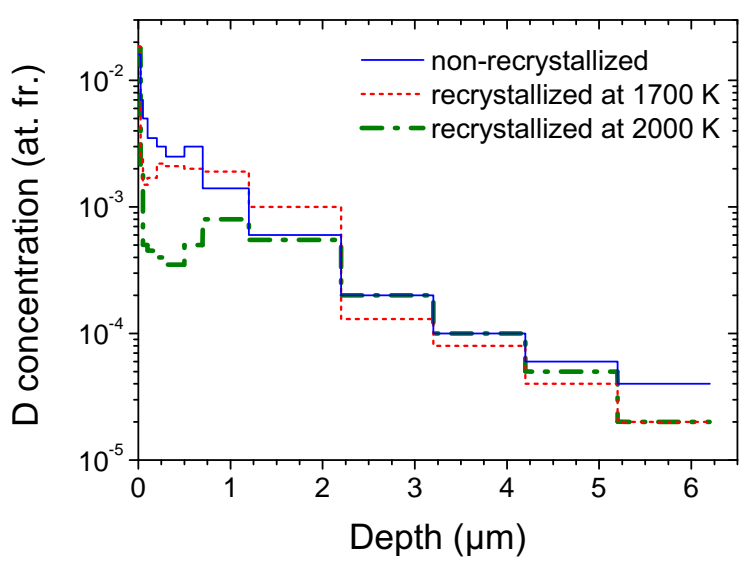

Fig. 3. NRA depth profiles of $\mathrm{D}$ retained in non-recrystallized and recrystallized tungsten samples after bombardment with $38 \mathrm{eV} / \mathrm{D}$ at a sample temperature of 300 K.

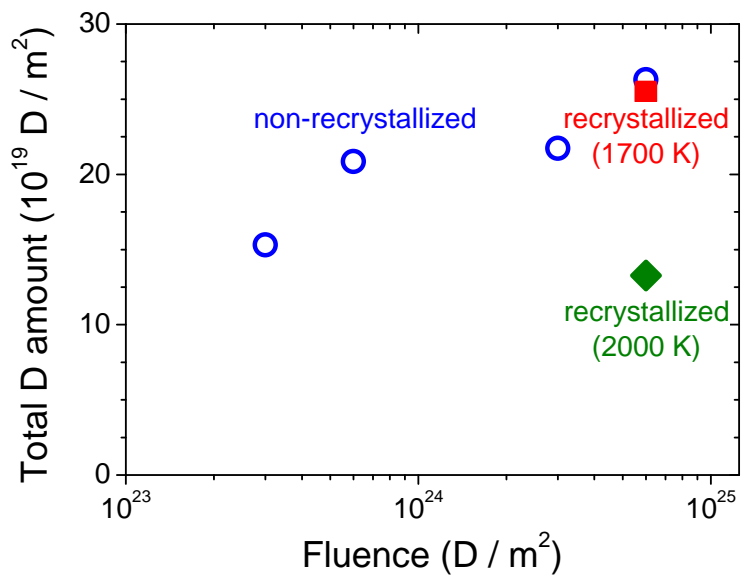

Fig. 4. Total amount of D retained in non-recrystallized and recrystallized tungsten samples after exposure to a deuterium plasma with $38 \mathrm{eV} / \mathrm{D}$ at a sample temperature of $300 \mathrm{~K}$ as determined by NRA. 


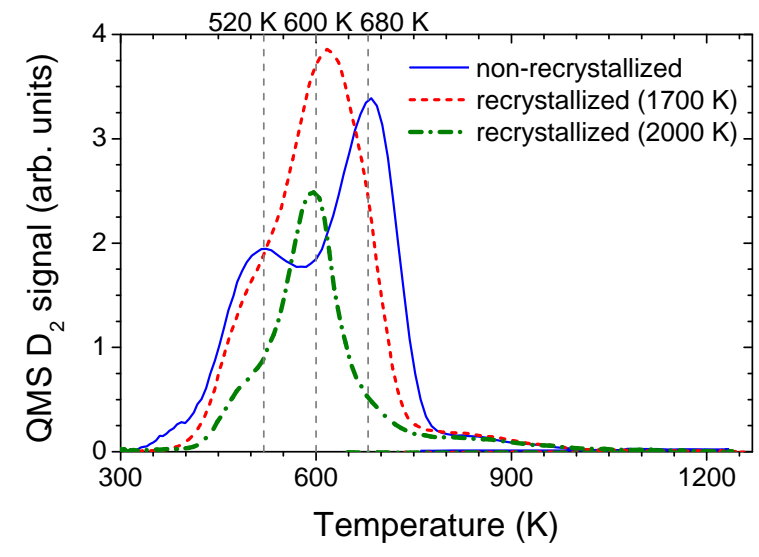

Fig. 5. $\mathrm{D}_{2}$ release spectra from non-recrystallized and recrystallized tungsten samples exposed to a fluence of $6 \times 10^{24} \mathrm{D} \mathrm{m}^{-2}$ with $38 \mathrm{eV} / \mathrm{D}$ at a sample temperature of $300 \mathrm{~K}$. The heating rate was $1.4 \mathrm{~K} / \mathrm{s}$ for all samples.
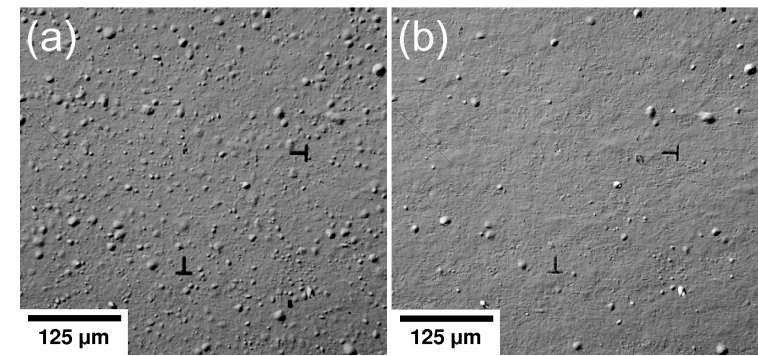

Fig. 6. Surface of non-recrystallized tungsten sample after implantation (a) and after TDS (b). T-shaped markers were applied to the sample surface by FIB. 